\title{
Academic Stress and Depression among Adolescents: A Cross-sectional Study
}

\author{
P JAYANTHI, *M THIRUNAVUKARASU AND "RAJAMANICKAM RAJKUMAR \\ From the Department of *Psychiatry, SRM Medical College Hospital \& Research Centre, SRM University, Chennai; and \\ Department of ${ }^{\#}$ Community Medicine, Meenakshi Medical College Hospital \& Research Institute, Enathur, Kanchipuram; Tamil \\ Nadu, India.
}

Correspondence to:

P Jayanthi, Research Scholar, SRM University, Chennai, India. jayanthiarul2011@gmail.com

Received: August 20, 2014;

Initial review: September 15, 2014;

Accepted: December 24, 2014.
Objective: To examine the relationship between academic stress and depression among adolescents.

Methods: A cross-sectional study was conducted at higher secondary schools in Tamil Nadu. 1120 adolescents were included in the study after screening by MINI-kid tool. Modified Educatonal Stress Scale for Adolescents was administered to all children.

Results: Adolescents who had academic stress were at 2.4 times (95\% $\mathrm{Cl}=0.9-2.4$ ) $(P<0.001)$ higher risk of depression than adolescents without academic stress.

Conclusions: Adolescents with severe academic stress need to be identified early as interventions to reduce academic stress is likely to affect the occurrence and severity of depression.

Keywords: Children, Mental health, School health.
A dolescence is a transitional stage from childhood to adulthood and is a time of major changes in all areas of functioning. Children and adolescents can experience various life stresses ranging from catastrophic or traumatic life events, persistent strain and daily hassles. Academic matters are the most important sources of chronic and sporadic stress for young people in both Western and Asian countries, and has significant associations with mental health problems, such as depression, anxiety and suicidal ideation $[1,2]$.

There is an increasing concern regarding study pressure and its relationships with mental health problems among school children and adolescents in India. Suicide is the third leading cause of death among adolescents, and unrevealed depression is a major cause. Academic stress may be contributing factor in depression. This study aimed to explore the relationship between depression and academic stress.

\section{Methods}

A case-control study was conducted at selected higher secondary schools in Tamil Nadu, between July 2013 and January 2014. Ethical approval was obtained from the Institutional Ethics Review Board and formal permission was obtained from the Chief Education Officer, Thiruvallur District and the Principals of the selected private schools. The samples were recruited from three private and one government higher secondary school.

A total of 2432 school-going adolescents studying in class 9th to 12th were screened using MINI-Kid [3,4], which is a screening tool for depression. 640 students who scored highly in the MINI-kid were subjected to further assessment by the Psychiatrist, who confirmed their depressive status. 612 students from this high-scoring group were enrolled as cases whereas 612 students who had a low score in MINI-kid were enrolled into the control group. Prior to data collection informed consent form was handed over to these 1224 students; 1198 parents gave their written informed consent. Finally 1120 adolescents (560-cases and 560-control group) were confirmed as the study sample.

Accompanying Editorial: Pages 202-203.

Beck Depression Inventory (BDI) [5] a psychometrically sound measure for screening depression among adolescents [6], was administered to the Case group. BDI was used specifically to classify the these adolescents into minimal, mild, moderate and severe depression. Modified Educational Stress Scale for Adolescents [7] was administered to both the Case and Control groups. The scale is based on the Ziandong Sun's educational stress scale for adolescents, which includes 6 dimensions and 30 items. This scale had 20 items and 6 dimensions. The Educational Stress Scale for Adolescents 


\section{What ThIS StUdy AdDS?}

- Academic stress is associated with depression among adolescents.

- Self-expectations and perceived pressure are the main causes for academic stress.

is considered a suitable measure for school-based mental health research in Asia [8]. In the present study, reliability score for Beck Depression Inventory was 0.85, and for Modified Educational Stress Scale was 0.81.

Statistical analysis: Statistical analysis was performed using the Statistical Package for Social Sciences Programme (SPSS) version 17.0. Descriptive statistics and inferential statistics such as Student's independent ttest, Karl Pearson correlation coefficient, Chi square test, Odds Ratio, and Multivariate logistic regression was used for analysis.

\section{Results}

Of the 612 adolescents diagnosed with depression during the study period, $45.7 \%$ had moderate, $25.4 \%$ had mild, $19.6 \%$ had severe, and $9.3 \%$ had minimal depression as per BDI.

In Case group, the mean (SD) academic stress score was 78.4 (4.67) whereas in Control group it was 41.9 (5.61). The percentage difference (95\% CI) score between case and control was 36.5\% (35.9\%-37.1\%) $(P<0.001)$.

A moderate positive relationship was found between the level of depression and the level of academic stress. The r value was 0.52 at $P<0.001$ level (Fig. 1).

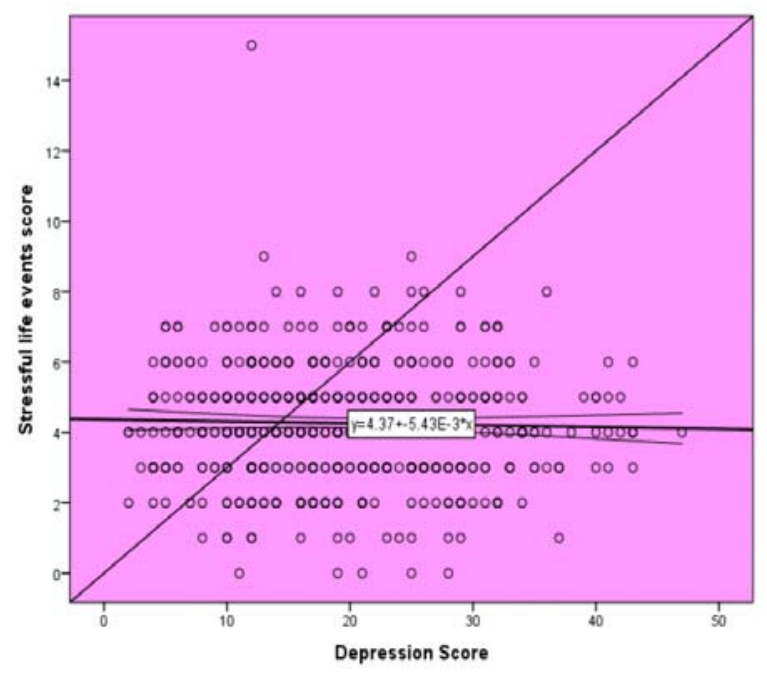

Fig. 1 Scatter plot shows the positive correlation between depression score and stressful life events score.
Adolescents with academic stress were found to have 2.4 times (95\% CI=0.9-2.4) $(P<0.001)$ more risk of having depression than the adolescents without academic stress. Demographic variables such as late adolescents, girls, XII standard, Tamil medium, and government school students had significant association with academic stress in Case group.

\section{Discussion}

The study findings revealed that the overall mean academic stress score difference between case and control group was 36.5. In case group $75 \%$ of the adolescents experienced severe academic stress whereas in control group $87 \%$ of the adolescents experienced mild academic stress.

These findings were consistent with previous studies [1], which found academic achievement to be significantly correlated with depression and Selfexpectations. Parent and teachers expectations were the main sources of academic stress among adolescents. Adolescent girls had higher academic stress than boys, similar to a previous Indian study [9].

The current study is limited because of adolescents sickness, absenteeism, unwillingness, incomplete questionnaires, and lack of matching. The findings implied that academic stress is associated with depression among adolescents. Adolescents with severe academic stress have to be identified early, and prompt interventions will likely prevent or ameliorate depression. Students should be counselled for stress factor by school counsellor or school heath nurse. Teachers should try to remove academic stress generating factors from learning environment by taking appropriate steps.

Contributors: JP: Conceptualisation of the study, collection, analysis of the data, writing the manuscript, finalised the manuscript and will act as the guarantor of the paper; TM: Conceptualisation of the study, edited, and critically evaluated the manuscript; RR: Writing the manuscript, edited, and critically evaluated.

Funding: None; Competing interests: None stated.

\section{REFERENCES}

1. Anderman EM. School effects on psychological outcomes during adolescence. J Edu Psycho. 2002;94:795-805.

INDIAN PEDIATRICS 
2. Ang RP, Huan VS. Relationship between academic stress and suicidal ideation: Testing for depression as a mediator using multiple regressions. J Child Psychi Human Develop. 2006;37:133-43.

3. Sheehan D, Shytle D, Milo K, Lecrubier Y, Hergueta T. M.I.N.I. - Kid Mini International Neuropsychiatric Interview for children and adolescents. 2005; Jan 1.p.6.

4. Reliability and validity of the MINI International Neuropsychiatric Interview for children and adolescents. Available from: http://clinicaltrials.gov/ct2/. Accessed September 12, 2014.

5. Beck AT, Steer RA, Garbin MG. Psychometric properties of the Beck Depression Inventory: Twenty five years of evaluation. Clinical Psycho Review. 1988;8:77-100

6. Basker M, Moses PD, Russell S, Swamidhas P, Russell S.
The psychometric properties of Beck Depression Inventory for adolescent depression in a primary care paediatric setting in India. J Child Adol Psychiatry Mental Health. 2007;1:8.

7. Sun, Jiandong, Dunne, Michael P, Hou, Xiang-Yu, et al. Educational Stress Scale for Adolescents: Development, validity, and reliability with Chinese students. J Psychoedu Assessment. 2011;29:534-46.

8. Truc TT, Loan KX, Nguyen ND, Dixon J, Sun J, Dunne MP. Validation of the Educational Stress Scale for Adolescents (ESSA) in Vietnam. Asia-Pacific J Public Health. 2012 Apr 11. [Epub ahead of print]

9. Deb S, Strodl E, Sun J. Academic- related stress among private secondary school students in India. J Asian Educa Develop Studies. 2014;3:118-34. 